\title{
BAYESIAN PERSPECTIVE-PLANE (BPP) FOR LOCALIZATION
}

\author{
Zhaozheng $\mathrm{Hu}^{1,2}$ and Takashi Matsuyama ${ }^{1}$ \\ ${ }^{I}$ Graduate School of Informatics, Kyoto University, Sakyo-Ku, Kyoto, Japan \\ ${ }^{2}$ College of Information Science and Technology, Dalian Maritime University, Dalian, China \\ $\{h u, t m\} @ v i s i o n . k u e e . k y o t o-u . a c . j p$
}

Keywords: Vision-based Localization, Bayesian Perspective-plane (BPP), Plane Normal, Maximum Likelihood.

\begin{abstract}
The "perspective-plane" problem proposed in this paper is similar to the "perspective-n-point (PnP)" or "perspective-n-line $(\mathrm{PnL})$ " problems, yet with broader applications and potentials, since planar scenes are more widely available than control points or lines in practice. We address this problem in the Bayesian framework and propose the "Bayesian perspective-plane (BPP)" algorithm, which can deal with more generalized constraints rather than type-specific ones to determine the plane for localization. Computation of the plane normal is formulated as a maximum likelihood problem, and is solved by using the Maximum Likelihood Searching Model (MLS-M). Two searching modes of 2D and 1D are presented. With the computed normal, the plane distance and the position of the object or camera can be computed readily. The BPP algorithm has been tested with real image data by using different types of scene constraints. The 2D and $1 \mathrm{D}$ searching modes were illustrated for plane normal computation. The results demonstrate that the algorithm is accurate and generalized for object localization.
\end{abstract}

\section{INTRODUCTION}

Vision-based localization has many applications in computer vision, robotics, augmented reality (AR), simultaneous localization and mapping (SLAM), and photogrammetric communities (Hartley \& Zisserman, 2004; Desouza et al., 2002; DurrantWhyte \& Bailey, 2006; Cham et al., 2010). The basic objective is to compute the position of the object or the camera in a reference coordinate system by using either multiple (two or more) or single image(s). For example, stereo vision is a commonly used approach for localization from two images, which can retrieve the $3 \mathrm{D}$ coordinates or position of an object in the scene from image disparity, baseline width, and camera focal length (Hartley \& Zisserman, 2004). Localization from single view usually relies on "prior knowledge" of the scene. In the literatures, many localization algorithms were developed by utilizing specific scene constraints, such as well-defined landmarks, natural or artificial objects, and the abstract points or lines, etc. For example, landmarks are commonly used for localization (Adan, 2010). Face position can be computed by referring to a 3D face model (Sun et al., 2008). Single image of a window in the street is possible to localize the camera (Johansson \& Cipolla, 2002). Camera pose can also be computed from corner structure (Shi et al., 2004). Besides these particular objects, many researches investigate the localization problem from a set of abstract points or lines, namely known as the perspective-n-point $(\mathrm{PnP})$ and perspective-n-line $(\mathrm{PnL})$ problems (Wolfe et al., 1991; Kneip et al., 2011; Hu \& Matsuyama, 2011). Besides points and lines, the position can also be computed from a plane or planar object. Planar structure, i.e., the plane normal and distance, can be computed from parallel lines, homography, conics, control points, textures, etc. For example, Hu et al determine the support plane from the geometric constraints of three control points $(\mathrm{Hu} \&$ Matsuyama, 2011). With four or more planar points, we can compute the homography and determine the plane position by decomposition (Zhang, 2000). The plane normal is also computed from vanishing line, if two sets of parallel lines are available in the scene (Lee et al., 2009). Other methods to compute the plane by using conics, reference lengths, textures, etc., are also reported in (Hartley \& Zisserman, 2004; Guo \& Chellappa, 2010; Witkin, 1981).

Existing methods for localization and planar structure computation from single view are mostly based on specific scene constraints. These methods are not generalized or practical enough. On the one hand, some strict constraints, which are required by the type-specific algorithm, are not available in the 
scene. On the other hand, some constraints, which are present in the scene, are difficulty to be utilized by the type-specific algorithm. In this paper, we propose the "perspective-plane" problem and address it by the Bayesian perspective-plane (BPP) algorithm. The proposed algorithm can utilize more generalized planar and out-of-plane constraints to determine the plane for localization.

\section{FORMULATION OF GEOMETRIC CONSTRAINTS}

From projective geometry, a physical plane and its image are related by a $3 \times 3$ homography matrix $H$ as follows (Hartley \& Zisserman, 2004; Zhang, 2000)

$$
X \cong H^{-1} x
$$

where $\cong$ means equal up to a scale, $x$ and $X$ are the coordinates for the image and physical point, respectively. The homography is computed from the plane normal and distance according to stratified reconstruction theories (Hartley \& Zisserman, 2004). With the recovered 2D coordinates, more planar geometric attributes, such as coordinate, distance, angle, length ratio, curvature, shape area, etc., are calculated readily. A metric reconstruction of the plane is also feasible with known plane normal and unknown distance. However, the absolute geometric attributes, such as coordinates, distance, etc., are equal to the actual ones up to a common scale. And non-absolute geometric attributes, such as length ratio, angle, etc., are equal to the actual ones.

Some out-of-plane geometric attributes can also be computed with a reference plane. For example, an orthogonal plane can be measured by referring to a known plane (Wang et al., 2005). If the vanishing line of a reference plane and one vanishing point along a reference direction are known, object height, distance, length ratio, angle, and shape area on the parallel planes etc., are readily computed (Criminisi et al., 2000). From projective geometry, both the vanishing line and the vanishing point along the normal are computed from the plane normal and the calibration matrix. Also, if the plane distance is unknown, those absolute out-of-plane geometric attributes are computed up to a common scale, while the non-absolute ones are equal to the actual ones.

Hence, we use $Q_{i}(N)$ to denote the $i^{\text {th }}$ geometric attribute, computed from the plane normal $N$. Scene prior knowledge can be formulated into constraints on plane normal. Let $u_{i}$ be the deterministic value of the geometric attribute that we know a prior. The measurement error is defined as

$$
d_{i}(N)=Q_{i}(N)-u_{i}
$$

By forcing zero measurement error, we can derive one geometric constraint on the plane normal

$$
C_{i}: d_{i}(N)=Q_{i}(N)-u_{i}=0
$$

Eq. (3) holds for both planar and out-of-plane constraints. Planar constraints are derived from prior knowledge of planar features, while the out-of-plane constraints are from the out-of-plane ones. The planar features are those that lie on the plane, such as the point, line, angle, curve, shape, etc., while the other are defined as out-of-plane features, such as an orthogonal line, an angle on another plane, etc. In a similar way, we can derive a set of geometric constraints as follow

$$
C=\left\{C_{i} \mid d_{i}(N)=Q_{i}(N)-u_{i}=0\right\}(i=1,2, \cdots, M)
$$

\section{BAYESIAN PERSPECTIVE-PLANE (BPP)}

\subsection{Localization from a Known Plane}

An arbitrary planar point can be localized from a known plane as follows

$$
\left\{\begin{array}{l}
X=\lambda K^{-1} x \\
N^{T} X=d
\end{array}\right.
$$

The first equation in Eq. (5) defines the backprojection ray, where the $3 \mathrm{D}$ point $\mathrm{X}$ lies (Hartley \& Zisserman, 2004). It can be determined from the camera calibration matrix and the image point. The second one is the plane equation with the normal $N$ and distance $d$. If we use the plane to expand a reference coordinate system, the camera can be localized as well (Zhang, 2000).

\subsection{Plane Normal from MLS-M}

\subsubsection{Maximum Likelihood Formulation}

The plane normal is assumed uniform distribution if there are no geometric constraint. The distribution changes from uniform to non-uniform, given one constrain. Distribution from a set of constraints is expected to have a dominant peak, from which the plane normal is solved. Therefore, computation of the plane normal $N$ is formulated as to maximize the following conditional probability 


$$
\underset{N}{\operatorname{argmax}} P\left(N \mid C_{1}, C_{2}, \cdots, C_{M}\right)
$$

By using Bayes' rule, Eq. (6) is transformed as

$$
P\left(N \mid C_{1}, C_{2}, \cdots, C_{M}\right)=\frac{P\left(C_{1}, C_{2}, \cdots, C_{M} \mid N\right) P(N)}{P\left(C_{1}, C_{2}, \cdots, C_{M}\right)}
$$

Assume that all the constraints are conditionally independent to each other so that we can derive

$$
P\left(C_{1}, C_{2}, \cdots, C_{M} \mid N\right)=\prod_{i=1}^{M} P\left(C_{i} \mid N\right)
$$

Assume uniform distribution of plane normal and we can substitute Eq. (8) into Eq. (7) to derive

$$
P\left(N \mid C_{1}, C_{2}, \cdots, C_{M}\right) \propto \prod_{i=1}^{M} P\left(C_{i} \mid N\right)
$$

In order to solve Eq. (6), we need to model $P\left(C_{i} \mid N\right)$, which is defined as the likelihood that the $i^{\text {th }}$ constraint is satisfied, given the plane normal $N$. We set the following rules to develop the model: 1) the likelihood is determined by the measurement error. More absolute measurement error yields lower likelihood. The maximum likelihood is reached if the measurement error is zero; 2) the maximum likelihoods (for zero measurement errors) for different constraints should be equal so that all constraints contribute equally; 3) the measurement error should be normalized to deal with the geometric attributes in different forms, units, and scales, etc. To meet these requirements, the normalized Gaussian function was proposed to model the likelihood as follows

$$
G\left(d_{i}(N) \mid u_{i}, \sigma_{i}\right)=\frac{1}{\sqrt{2 \pi} \sigma_{i}} \exp \left(-\frac{d_{i}^{2}(N)}{2 u_{i}^{2} \sigma_{i}^{2}}\right)
$$

The measurement error is normalized by $u_{i}$ from Eq. (3). Obviously, Eq. (10) satisfies the first rule. The likelihood also decreases with the absolute measurement errors. The maximum likelihood is reached at zero measurement error. In order to satisfy the second rule, the standard deviations for all Gaussian models should be equal: $\sigma_{i}=\sigma_{j}=\sigma$. Hence, the likelihood model is derived as

$$
P\left(C_{i} \mid N\right)=G\left(d_{i}(N)\right)=\frac{1}{\sqrt{2 \pi} \sigma} \exp \left(-\frac{d_{i}^{2}(N)}{2 u_{i}^{2} \sigma^{2}}\right)
$$

Substitution Eq. (11) into Eq. (9) yields

$$
P\left(N \mid C_{1}, C_{2}, \cdots, C_{M}\right) \propto \prod_{i=1}^{M} \exp \left(-\frac{d_{i}^{2}(N)}{2 u_{i}^{2} \sigma^{2}}\right)
$$

The plane normal is thus computed as

$$
\underset{N}{\arg \max } \exp \left(-\sum_{i=1}^{M} \frac{d_{i}^{2}(N)}{2 u_{i}^{2} \sigma^{2}}\right)
$$

\subsubsection{Searching for Maximum Likelihood}

A maximum likelihood searching model (MLS-M) is used to solve Eq. (13). Two searching modes of $2 \mathrm{D}$ and $1 \mathrm{D}$ are proposed in the following.

\section{a. 2D searching mode}

A unit normal corresponds to a point on the Gaussian sphere surface so that the searching space is defined. In practice, we search on the Gaussian hemisphere to reduce the searching space into half, because only the planes in front of the camera are visible in the image. A uniform sampling is realized by evenly partition Gaussian hemisphere, which can be solved by using the recursive zonal equal area sphere partitioning algorithm (Leopardi, 2004). The likelihood for each sample is computed so that the maximum likelihood is calculated by sorting, the corresponding plane normal is then determined. Since Gaussian sphere in 3D space is 2D, we call it a 2D searching mode.

\section{b. $1 D$ searching mode}

1D searching mode is feasible if one linear constraint is available in the scene. In practice, some linear constraints can be derived from parallel lines, orthogonal lines or planes, etc. We introduced the 1D searching mode by using orthogonal line as an example. Let $L$ be the orthogonal line, with the image $l$. Because $L$ is parallel to the plane normal, the vanishing point $V_{N}$ along the normal lies on $l$ and satisfy the following equation

$$
l^{T} V_{N}=l^{T}(K N)=\left(K^{T} l\right)^{T} N=0
$$

The normal $N$ also lies on Gaussian sphere so that

$$
\left\{\begin{array}{l}
\left(K^{T} l\right)^{T} N=0 \\
N^{T} N=1
\end{array}\right.
$$

Eq. (15) defines a circle in 3D space, which is the intersection of the plane with the Gaussian sphere. Therefore, we need to search on the circle (actually half of the circle). Similarly, a uniform sampling on the circle is required. The likelihood for each sample is computed. And the maximum likelihood is finally searched to solve the plane normal.

\subsection{Plane Distance Computation and Localization}

Assume unit plane distance and re-write Eq. (5) as 


$$
\left\{\begin{array}{l}
X^{\prime}=\lambda^{\prime} K^{-1} x \\
N^{T} X^{\prime}=1
\end{array}\right.
$$

Compared to Eq. (5), it can be derived that $X^{\prime} \cong X$. Hence, we can localize all the planar points in the camera coordinate system up to a common scale. In order to compute the actual coordinates, a reference length or distance is required. For example, the scale can be determined from the actual length $L_{A B}$ between two points $A$ and $B$ as follows

$$
\beta=\frac{L_{A B}}{\left\|X_{A}^{\prime}-X_{B}^{\prime}\right\|}
$$

where $X_{A}^{\prime}$ and $X_{B}^{\prime}$ are the $3 \mathrm{D}$ coordinates of two points that are computed from Eq. (16). With the computed scale factor, the actual plane distance is $d=\beta$. And the actual $3 \mathrm{D}$ position is

$$
X=\beta X^{\prime}
$$

\section{EXPERIMENTAL RESULTS}

The proposed algorithm was tested with two real image experiments. A Nikon D700 camera was used to generate the real images of $2218 \times 1416$ (pixel) resolution. It was calibrated with Zhang's calibration algorithm (Zhang, 2000) with 1369.2-pixel focal length and principle point at $[1079,720]^{\mathrm{T}}$ (pixel). The calibration results were used for planar structure computation and localization. As there are various scene constraints in practice, it is difficult to discuss all of them. We chose two representative constraint types of known angle and length ratios to validate the proposed model in the experiments.

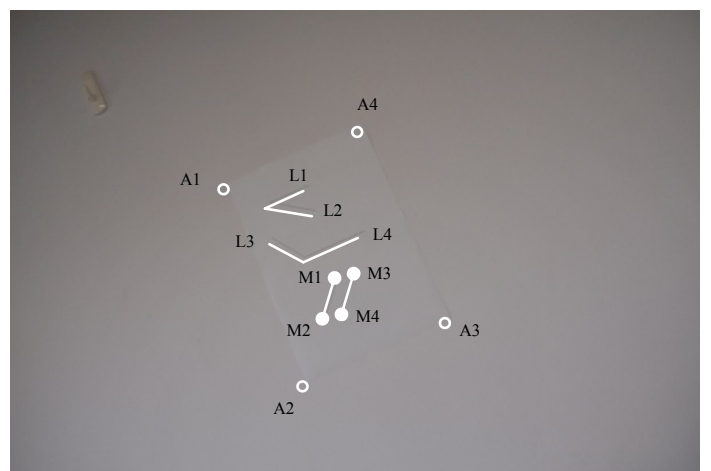

Figure 1: Three planar constrains (two known angles and one length ratio) from lines and points for localization.

The algorithm was tested with planar constraints first. Figure 1 shows an image of a white paper attached on the wall. Six line segments were drawn
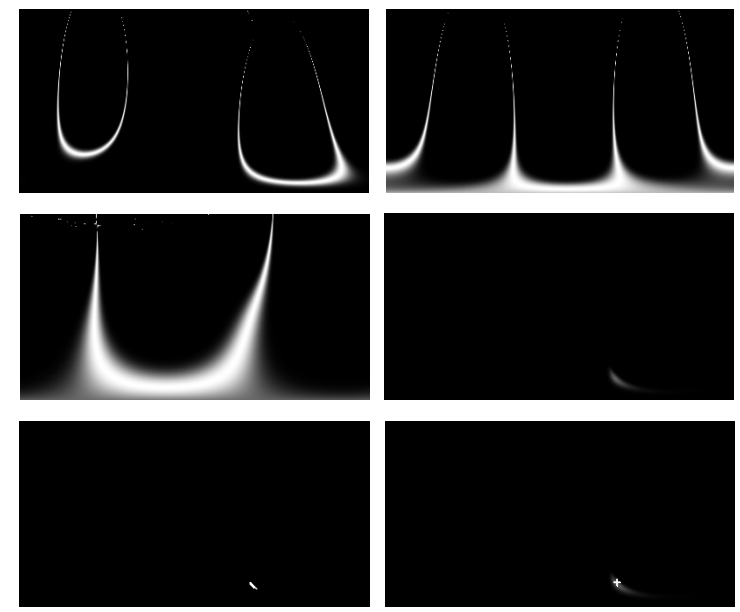

Figure 2: From upper left to lower right: (a) (b) (c) likelihoods from three geometric constraints, (d) the joint likelihood, (e) positions of the 30 normal of the highest likelihoods; (f) the computed normal marked by ' + '.

on the paper. We derived two angles (34.1 and 126.6 degrees) from L1-L2 and L3-L4, and one length ratio between the distances of M1-M2 and M3-M4 to derive three planar constraints. The $2 \mathrm{D}$ searching mode was applied then. The likelihoods from different geometric constraints are shown in Figure 2(a) (c), where the non-uniform distributions of the plane normal are observed. The joint likelihood is shown in Figure 2(d), where a bright area on the bottom is observed. Figure 2(e) shows the 30 plane normal with the highest likelihoods. The plane normal computed from the joint likelihood map is marked by ' + ' (see Figure 2(f)), with the result $\left[\begin{array}{llll}0.0993 & 0.1679 & 0.9808\end{array}\right]^{\mathrm{T}}$.

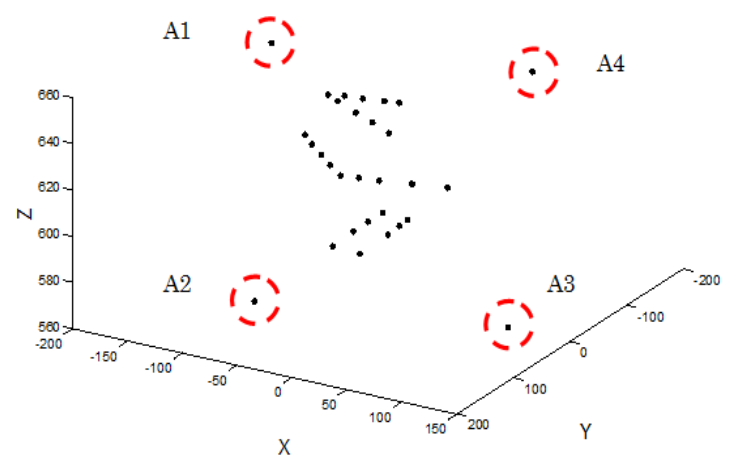

Figure 3: Localize points and lines in the camera coordinate system (unit in $\mathrm{mm}$ ).

The plane distance was computed by using the reference length of the white paper $(297 \mathrm{~mm})$. The points on the plane were localized. Figure 3 shows $3 \mathrm{D}$ positions of the points on the six line segments, and the four corners (A1 A4 marked by 'o'), all in 
the camera coordinate system. The computation results were validated as follow. The lengths of A2A3, A3-A4, and A1-A4 were computed from the recovered 3D coordinates as 206.7, 294.6, and 211.8 $(\mathrm{mm})$, respectively. Compared to ground truth data (the size of the white paper), the absolute errors are $3.3,2.4$, and $1.8(\mathrm{~mm})$, corresponding to $1.6 \%$, $0.8 \%$, and $0.9 \%$ relative errors. It demonstrates that the proposed model is accurate.

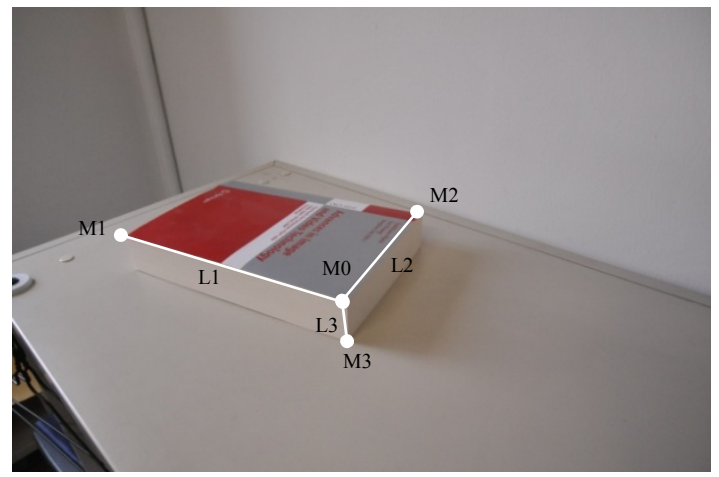

Figure 4: Localization from 3D corner constraints.

The algorithm was further tested with both planar and out-of-plane constraints by using 1D searching mode. As shown in Figure 4, a 3D corner structure of a book is defined by the three orthogonal lines of L1, L2, and L3. From these three lines, three planes are defined as L1-L2, L1-L3, L2-L3. For each plane, we derived two constraints: 1) one orthogonal line to the plane; 2) one right angle between the other two lines on the plane, as shown in Figure 4. They were used to localize each plane.
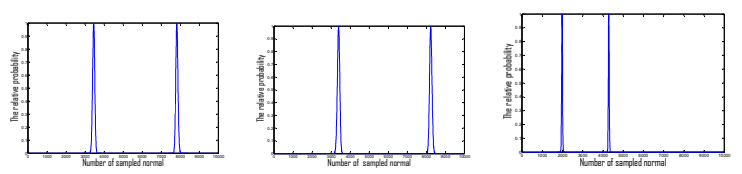

Figure 5: From left to right: likelihoods of the normal of the planes defined by: a) L1-L2, b) L1-L3, c) L2-L3.

The 1D searching mode was applied. The searching circles were defined from the orthogonal line constraints, on which 10,000 points were uniformly sampled, respectively. The likelihoods for each normal of the three planes were computed afterwards (see Figure 5(a) (c)). For each plane, two peaks are observed from the likelihood curve, which indicates two solutions (also see multiple solutions in (Shi et al., 2004)). Hence, eight combinations for the normal of the three planes are derived, two of which satisfy the mutually orthogonality constraints. In order to eliminate the ambiguity, we utilized one more constraint from the ratio of the length (M0-
M1) to the width (M0-M2) to uniquely determine the normal of the plane L1-L2. The likelihood from such constraint and the joint likelihood are illustrated in Figure 6 . In the joint likelihood, a unique peak is observed (see Figure 6(b)). From the joint likelihood, the maximum likelihood was searched and the normal to the L1-L2 plane was computed as $\left[\begin{array}{llll}-0.0022 & 0.8425 & 0.5387\end{array}\right]^{\mathrm{T}}$. With the computed L1-L2 normal, the normal for the other two planes were also uniquely determined.
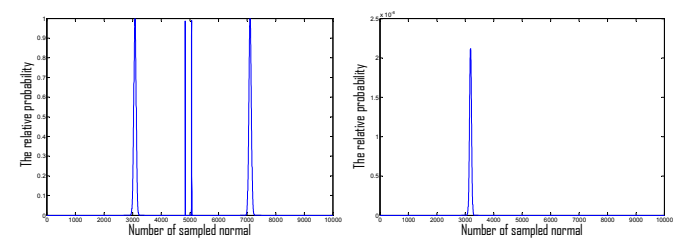

Figure 6: From left to right: a) likelihood from the length ratio constraint; b) joint likelihood from all constraints.

We used one actual length to compute the plane distance and localize the corner points in the camera coordinate system. Their 3D positions are shown in Figure 7. The camera was also localized in the reference coordinate system, defined by the corner structure, with M0 as the origin, and L1, L2, L3 as the three axes, where the camera position is [-21.64, $-23.82,-12.26]^{\mathrm{T}}$ (in $\mathrm{cm}$ ). Hence, the object and the camera were localized from each other. We validated the results as follows. The calculated $3 \mathrm{D}$ coordinates were used to fit the three lines. The lines are mutually orthogonal and have an angle of 90 degrees in between, which are used as ground truth. The computed angles in-between are 89.4, 88.1, and 94.1 degrees, respectively, with the absolute errors of $0.6,1.9$, and 4.1 degrees. The relative errors are $0.7 \%, 2.1 \%$, and $4.6 \%$, with the average $2.4 \%$. The results demonstrate that the algorithm is accurate.

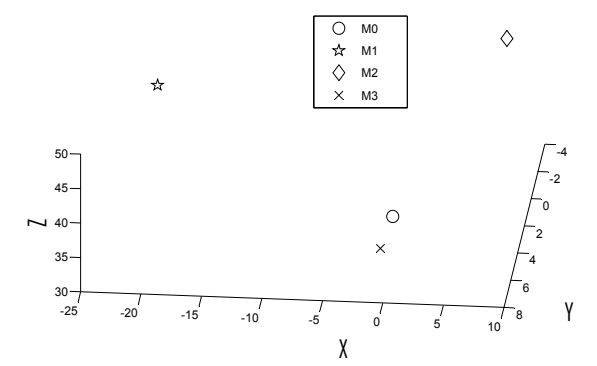

Figure 7: Localize the four corner points in the camera coordinate system (unit in $\mathrm{cm}$ )

\section{CONCLUSIONS}

In this paper, we proposed the Bayesian perspective- 
plane (BPP) algorithm to address the "perspectiveplane" problem, which can localize object/camera by determining planar structure from more generalized planar and out-of-plane constraints. Computation of the plane normal is formulated as a maximum likelihood problem and is solved by MLS-M. Both 2D and 1D searching modes were presented. The BPP algorithm has been tested with real image data. The results show that the proposed algorithm is generalized to utilize different types of constraints for accurate localization.

\section{ACKNOWLEDGEMENTS}

The work presented in this paper was sponsored by a research grant from the Grant-In-Aid Scientific Research Project (No. P10049) of the Japan Society for the Promotion of Science (JSPS), Japan, and a research grant (No. L2010060) from the Department of Education (DOE), Liaoning Province, China.

\section{REFERENCES}

Hartley, R., and Zisserman, A., 2004. Multiple view geometry in computer vision, Cambridge, Cambridge University Press, $2^{\text {nd }}$ Edition.

Desouza, G. N., and Kak, A. C., 2002. Vision for mobile robot navigation: a survey, IEEE Trans on Pattern Analysis and Machine Intelligence, 24(2): 237-267.

Durrant-Whyte, H. and Bailey, T., 2006. Simultaneous Localization and Mapping (SLAM): part I the essential algorithms, IEEE Robotics and Automation Magazine, 13 (2): 99-110.

Cham, T., Arridhana, C., Tan, W. C., Pham, M. T., Chia, L. T., 2010. Estimating camera pose from a single urban ground-view omni-directional image and a 2D building outline map, IEEE International Conference on Computer Vision and Pattern Recognition (CVPR), IEEE Press.

Adan, A., Martin, T., Valero, E., Merchan, P., 2009. Landmark real-time recognition and positioning for pedestrian navigation, CIARP, Guadalajara, Mexico,

Sun, Y., and Yin, L., 2008. Automatic pose estimation of 3D facial models, IEEE International Conference on Pattern Recognition (ICPR), IEEE Press.

Johansson, B., and Cipolla, R., 2002. A system for automatic pose-estimation from a single image in a city scene, In IASTED Int. Conf. Signal Processing, Pattern Recognition and Applications, Crete, Greece

Shi, F., Zhang, X., and Liu, Y., 2004. A new method of camera pose estimation using 2D-3D corner correspondence, Pattern Recognition Letters, 25(10): 805-809.

Wolfe, W., Mathis, D., Sklair, C., and Magee, M., 1991. The perspective view of three Points, IEEE
Transaction on Pattern Analysis and Machine Intelligence, 13(1): 66-73.

Kneip, L., Scaramuzza, D., and Siegwart, R., 2011. A novel parameterization of the perspective-three-point problem for a direct computation of absolute camera position and orientation, IEEE International Conference on Computer Vision and Pattern Recognition (CVPR), IEEE Press.

$\mathrm{Hu}, \mathrm{Z}$., and Matsuyama, T., 2011. Perspective-three-point (P3P) by determining the support plane, International Conference on Computer Vision Theory and Applications (VISAPP), SciTePress.

Zhang, Z., 2000. A flexible new technique for camera calibration, IEEE Transactions on Pattern Analysis and Machine Intelligence, 22(11):1330-1334.

Lee, D. C., Hebert, M., and Kanade, T., 2009. Geometric reasoning for single image structure recovery, IEEE International Conference on Computer Vision and Pattern Recognition (CVPR), IEEE Press.

Guo, F., and Chellappa, R., 2010. Video metrology using a single camera, IEEE Trans on Pattern Analysis and Machine Intelligence, 32(7): 1329 -1335.

Witkin, A. P., 1981. Recovering surface shape and orientation from texture, Artificial Intelligence, 17(1-3): 17-45.

Wang, G., Hu, Z., Wu, F., and Tsui, H., 2005. Single view metrology from scene constraints, Image and Vision Computing, 23(9): 831-840.

Criminisi, A., Reid, I., and Zisserman, A., 2000. Single view metrology, International Journal of Computer Vision, 40(2): 123-148.

Leopardi, P., 2006. A partition of the unit sphere into regions of equal area and small diameter, Electronic Transactions on Numerical Analysis, 25(12):309-327. 